\title{
Contrast Enhancement of Remote Sensing Images using DWT with Kernel Filter and DTCWT
}

\author{
Ruchika Mishra \\ LNCT, Bhopal
}

\author{
Utkarsh Sharma \\ Asst. Professor, \\ LNCT, Bhopal
}

\author{
Manish Shrivastava, $\mathrm{PhD}$ \\ HOD, \\ LNCT, Bhopal
}

\begin{abstract}
Image enhancement is the indispensable features in image processing to increase the contrast of the remote sensing data and to provide better transform representation of the remote image data. This paper presents a new method to improve the contrast and intensity of the image data. The method employs that the discrete wavelet transform with Kernel adaptive filtering. The performance of this algorithm is analysed and compared between EME and PSNR using simulator MATLAB 2009A.
\end{abstract}

\section{Keywords}

Brightness Preservation, Histogram Equalization, Discrete Wavelet Transform, Dual Tree Complex Wavelet Transform, Kernel filter

\section{INTRODUCTION}

Digital image processing is a widely used area under discussion now a days and it is typically used in the area of computer science and mathematics employ procedures which can be mathematically difficult, but fundamental idea behind digital image processing is quite easy. The critical mean of image processing is to use data enclosed in the image to enable the system to comprehend, identify and interpret the developed information obtainable from the image pattern [1].

Image Enhancement is one of the most significant and complicated techniques in image research. The aim of image enhancement is to get better the visual appearance of an image, or to provide a "better transform illustration for upcoming automated image processing. Several images like satellite images, medical images, aerial images and still real life photographs undergo from poor contrast and noise. It is essential to enhance the contrast and remove the noise to increase image quality. One of the most important stages in medical images detection and analysis is Image Enhancement techniques which improve the quality (clarity) of images for human viewing, abolish blurring and noise, increasing contrast, and revealing facts are examples of enhancement operations. The improvement technique differs from one field to another according to its objective [2]. By unscrambling smooth and detail areas of an image, the algorithm is applied to each of them to avoid excessive enhancement of noises. In some cases, quality of images is influenced by environment medium and water medium, consequently image enhancement is mandatory. Image enhancement (IE) has contributed to research advancement in a variety of fields. Some of the areas in which IE has wide applications such as: in forensic, atmospheric science and medical imaging etc. There are various image enhancement methods that have been proposed and developed, the most accepted method being Histogram Equalization. It usually increases the global contrast of the images mostly in cases where the important and useful data of the image is shown by low contrast values. Histogram equalization is a simple and effective contrast enhancement technique which distributes pixel values uniformly such that enhanced image have linear cumulative histogram. The HE technique is a global operation therefore; it does not defend the image brightness. In this paper, it uses DWT with kernel adaptive filtering which greatly increase the contrast and overcome the problem of HE.

There are many image enhancement techniques that have been proposed and developed, the most popular method being Histogram Equalization. This technique is one of the most popular methods for image enhancement due to its simplicity and efficiency. It usually increases the global contrast of the images mostly in cases where the important and useful data of the image is shown by low contrast values. The image enhancement methods can mostly divided in to the following two categories:

\section{$>$ Spatial Domain Methods \\ $>$ Frequency Domain Methods}

\subsection{Spatial domain methods}

The spatial domain method [3] method directly manipulates the image data array, either by point processing or area processing. Basically it deals with spatial frequency, i.e. difference between the highest and the lowest values of a contiguous set of pixels. The approaches regarding image enhancement using spatial domain methods can be divided into two categories global image enhancement and local image enhancement. Global methods are mainly histogram modifications that aim to exploit the full dynamic range of a rendering device by modifying the histogram of an image. The attractiveness is their simplicity and minor computational effort. However it is often necessary to enhance detail over a smaller area. So, the local image enhancement method plays a major role in those applications. This method can be characterized by equation 1 .

$$
\mathrm{G}(\mathrm{x}, \mathrm{y})=\mathrm{T}[\mathrm{F}(\mathrm{x}, \mathrm{y})]
$$

Where $F(x, y)$ is input image, $G(x, y)$ is output image and $T$ is an operator on $f$, defined over some locality of $f(x, y)$.

The idea of blurring an image by reducing its high frequency components or sharpening an image by increasing the magnitude of its high frequency components is intuitively easy to comprehend. Conversely, computationally, it is recurrently more efficient to implement these operations as convolutions by small spatial filters in the spatial domain.

\subsection{Frequency domain methods}

In the frequency domain [4] [5] relation the discrete convolution is often more efficiently the using fast Fourier transform algorithm. In a typical image enhancement problem $\mathrm{f}$ 
$(\mathrm{x}, \mathrm{y})$ is given and the goal after computation of $\mathrm{F}(\mathrm{u}, \mathrm{v})$ is to select $\mathrm{H}(\mathrm{u}, \mathrm{v})$ so that the desired image given by:

$\left.\mathrm{G}(\mathrm{x}, \mathrm{y})=\mathrm{F}^{-1}\{\mathrm{H}(\mathrm{u}, \mathrm{v}) * \mathrm{~F}(\mathrm{u}, \mathrm{v}))\right\}$

It also exhibits some highlighted attribute of $\mathrm{f}(\mathrm{x}, \mathrm{y})$ for example ends in $\mathrm{f}(\mathrm{x}, \mathrm{y})$ can be emphasize by using a function $\mathrm{H}(\mathrm{u}, \mathrm{v})$ which emphasizes the high frequency components of $\mathrm{F}$ $(\mathrm{u}, \mathrm{v})$.

The goal of image enhancement techniques is to develop a quality or contrast of an image such that enhanced image is better than the real image. Several image enhancement techniques have been proposed in both spatial and frequency domains. The rest part of research paper is organized in this way; the section 2 describes the previous work done. Section 3 gives detailed description of enhancement technique. Section 4 describes the proposed methodology. Section 5 shows the results produced by the analysis of proposed work and last section concluded about the research paper and its future scope.

\section{RELATED WORK}

This section describes some previous works done in contrast enhancement of the remote sensing image data in the literature:

G.Veena, V.Uma, Ch. Ganapathy Reddy [6] proposed an algorithm in which it first performs the DWT to decompose the input image into a set of band-limited components, called $\mathrm{HH}$, HL, LH, and LL sub bands. Since the LL sub band has the illumination information, the log-average luminance is calculated in the LL sub band for computing the dominant brightness level of the input image The LL sub band is divided into three low, middle, and high concentration layers according to the principal intensity level. The adaptive intensity transfer function is deliberated in three partitioned layers by the foremost intensity level, the knee transfer function, and the gamma alteration function. Subsequently, the adaptive transfer function is concerned for colour preserving high quality contrast enhancement. The resultant enhanced image is obtained by the inverse DWT (IDWT).

Chi-Farn Chen, Hung-Yu Chang, Li-Yu Chang [7] developed a fuzzy based approach to contrast enhancement of the remote sensing image data to partition the image pixel values into dissimilar degrees of associates in order to reimburse the local brightness lost in the dark and bright areas. The algorithm includes three steps: primarily, the satellite image is distorted from gray-level space to membership space by Fuzzy c- Means clustering. Secondly, suitable stretch model of each cluster is constructed based on corresponding memberships. Third, the image is changed back to the graylevel space by merging stretched gray values of each cluster

Deepak Kumar Pandey, Rajesh Nema [8] proposed a method to improve the quality of image using Kernel Padding and DWT with Image Fusion that enhances the contrast of Images that has varying intensity distribution particularly satellite images, maintain the brightness of images, sharpens the edges and abolish the blurriness of images. Fundamentally this is a pixel based edge guided image fusion technique. In this technique LL sub band of Image DWT is processed by contrast enhancement section where based on image brightness level image is decomposed in different layers and then every layers intensity is stressed or compressed by generating intensity transformation function. The partitioned intensity layers are also processed by canny edge detection method as all the satellite images includes the noise due to atmospheric turbulence and this is Gaussian by nature. The Canny edge detector is the best method for detecting edges of image in the existence of Gaussian noise. At last the contrast enhanced images are fused according to the weight map determined byedge map of image

Demirel [9] proposed a new method for enhancement of satellite images contrast. Their method was based on Discrete Wavelet Transform (DWT) and singular-value decomposition. They first applied DWT to input image to divide it into four frequency sub-bands, then used singular value decomposition and then again applied inverse DWT to reconstruct the image. This technique showed enhanced results than conventional Brightness preserving Dynamic Histogram Equalization (BPDHE) method and other methods.

Eunsung Lee [10] proposed the method which uses dominant brightness level of Image for decomposing the Image in different three layers and then these layers are used for appraisal of adaptive intensity transfer function. This predictable adaptive intensity transfer function is used for image contrast enhancement subsequently these layers are fused to get enhanced image.

Shujun Fu , Qiuqi Ruan, Wenqia Wang [11] presented a robust inverse diffusion equation method which sharpens image details by a robust Laplacian after demonstrating the equivalence of the sharpening by the Laplacian to inverse heat equation processing. Image gradient magnitude is used to avoid the noise magnification. At the same time, the min-mod function is used to manage diffusion flux adaptively, which reduces effectively overshoots inherent in the Laplacian. The Experimental results demonstrate that this algorithm can enhance important details of image data effectively exclusive of overshoots, giving the opportunity for a good interpretation and subsequent processing.

Artur Loza, David R. Bull, Paul R. Hill, Alin M .Achim [12] proposed a new method for contrast enhancement of images and image sequences of low-light or unevenly illuminated scenes based on statistical modeling of wavelet coefficients of the image .A non linear enhancement function has been designed based on the local dispersion of the wavelet coefficients modeled as a bivariate Cauchy distribution inside the same statistical framework, a synchronized noise reduction in the image is presented by means of a shrinkage function, therefore preventing noise strengthening. The projected enhancement method has been publicized to execute very well within sufficiently lighted up and noisy imagery, better performing than the other ordinary methods, in provisions of contrast enhancement and noise go down in the output data.

Adin Ramirez Rivera, Byungyong Ryu, and Oksam Chae [13] proposed a content-aware algorithm that improves the dark images, sharpens edges, expose details in textured regions, and conserve the smoothness of flat regions. This algorithm generates an ad hoc transformation for every image, adjusting the mapping functions to each image characteristic to produce the maximum enhancement. They examined the contrast of the image in the boundary and surface regions, and cluster the information with common characteristics. These clusters model the relations within the image, by which the transformation functions were extracted. The outcomes were then adaptively united, by considering the human vision system characteristics, to boost the details in the image. This method improves the manifestation of human features, blue skies with or without shades without initiating object. Conversely, it is unable to reobtain the information from the shadowed or dark areas of images that had near-black intensities. 


\section{IMAGE ENHANCEMENT TECHNIQUE}

The quality of images can be improved by using various enhancement technique and several techniques has been developed so far. This dissertation illustrates the several methods of image enhancement or to improve the quality of image.

\subsection{Discrete Wavelet Transform}

These days, wavelets have been used quite frequently in image processing. It is used for feature extraction, de-noising, compression, face detection, image super resolution. The decomposition of images into dissimilar frequency ranges permits the isolation of the frequency components introduced by "intrinsic deformations" or "extrinsic factors" into definite sub bands. This procedure results in isolating small changes in an image mainly in high frequency sub band images. Therefore discrete wavelet transform (DWT) [6] is an appropriate tool to be used for designing a classification system. The 2-D wavelet decomposition of an image is performed by applying 1-D DWT beside the rows of the image first, and, after that, the results are decomposed beside the columns. Such operation results in four decomposed sub band images referred to as lowlow (LL), low-high (LH), high-low (HL), and high-high (HH). Where, the signal is denoted by the sequence $\mathrm{CAj}$, where $\mathrm{Aj}$ is an integer. The low pass filter is denoted by Lo_D while the high pass filter is denoted by Hi_D. At each level, the high pass filter build detail information, whereas the low pass filter associated with scaling function generates coarse approximations. On every decomposition level, the half band filters produce signals spanning only half the frequency band. It doubles the frequency resolution as the vagueness in frequency is decreased by half. The frequency components of those sub-band images cover the frequency components of the novel image as shown in Figure 3.1 (a)

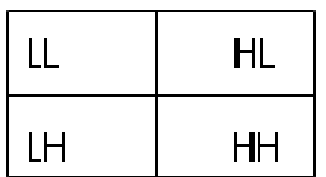

Figure 3.1(a) Result of 2-D DWT

Comprehensible decomposition steps of Discrete Wavelet Transform as shown here in Figure 3.1 (b).
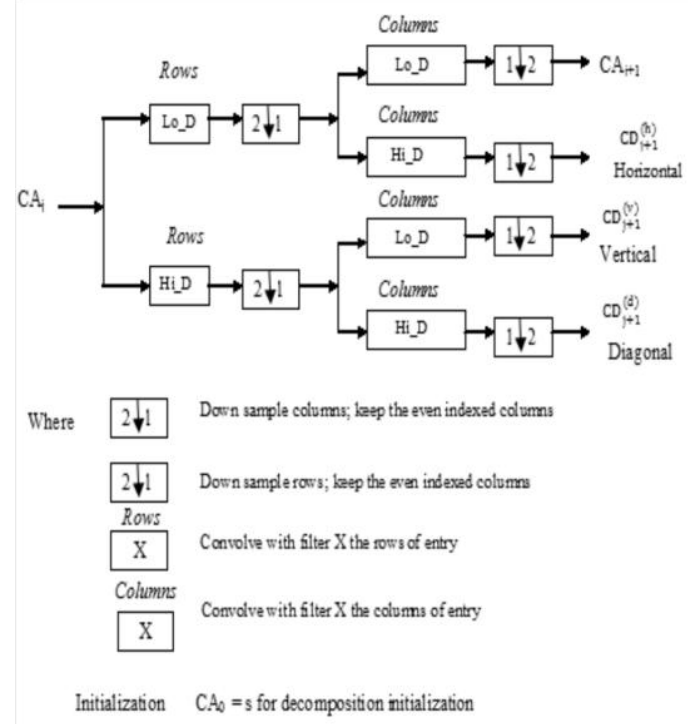

Figure 3.1(b) 2D-DWT Functional Process.

\subsection{Local Enhancement}

Previous methods of histogram equalizations and histogram matching are global. Hence, local enhancement [14] is used. Illustrating the square or rectangular neighbourhood (mask) and move the centre from pixel to pixel. For every neighbour, determine histogram of the points in the neighbourhood. Find the histogram equalization/specification function. Map gray level of pixel centred in neighbourhood. It can utilize new pixel values and preceding histogram to calculate next histogram.

\subsection{Contrast Stretching}

To expand the range of brightness values in an image the contrast enhancement techniques are used, so that the image can be efficiently displayed in a manner desired by the analyst. The level of contrast in an image may vary due to poor illumination or inappropriate setting in the acquisition sensor device. Consequently, there is a need to manipulate the contrast of an image in order to reimburse for difficulties in image achievement. The idea behind contrast stretching is to increase the dynamic range of the gray levels in the image being processed. The idea is to modify the dynamic range of the grey-levels in the image. Linear Contrast Stretch is the simplest contrast stretch algorithm that stretches the pixel values of a low-contrast image or high-contrast image by extending the dynamic range across the whole image spectrum from $0-(\mathrm{L}-1)$.

\subsection{Histogram Processing}

Histogram processing is used in image enhancement the information inherent in histogram can also used in other image processing application such as image segmentation and image compression. A histogram simply plots the frequency at which each grey-level occurs from 0 to 255 or (black -white). Histogram processing should be the initial step in preprocessing. To produce a much better image histogram equalization and histogram specification (matching) are two methods widely used to modify the histogram of an image.

The histogram is a discrete function that is shown in figure 3.4 Histogram correspond to the frequency of happening of all gray level in the image, which means it notified us how the values of individual pixel in an image are dispersed. Histogram is given as:

$\mathrm{h}(\mathrm{rk})=\mathrm{nk} / \mathrm{N}$

Where $r_{k}$ and $n_{k}$ are intensity level and number of pixels in image with intensity $r_{k}$ respectively.

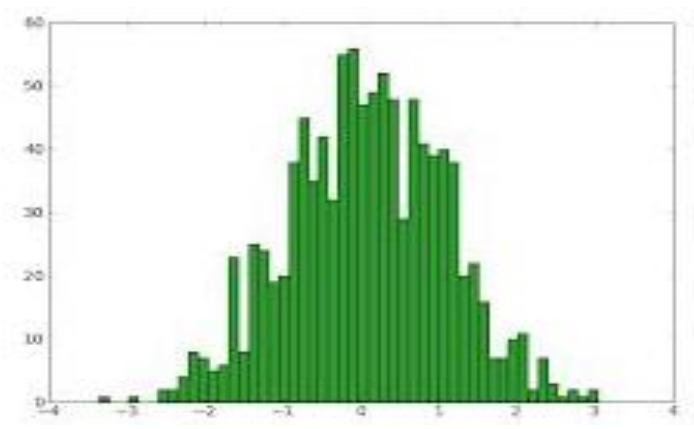

Figure 3.4 Histogram

Histogram equalization [15] is a frequent used technique for enhancing the quality of images. Assume we have an image which is primarily dark. Then its histogram would be skewed in the direction of the lower end of the grey scale and all the image detail is compressed into the dark end of the histogram. 
If we could 'stretch out' the grey levels at the dark end to produce a more uniformly distributed histogram then the image would become much clearer. Histogram equalization stretches the histogram across the entire spectrum of pixels $(0-255)$. It increases the contrast of images for the finality of human inspection and can be applied to normalize illumination variations in image understanding problems. Histogram equalization is one of the operations that can be applied to obtain new images based on histogram specification or modification. Histogram equalization is considered a global technique.

\section{PROPOSED METHODOLY}

For improving the quality or contrast of image there are several techniques has been developed but in this paper we proposes a novel method to increase the contrast of the remote sensing image data. This technique includes DWT with kernel adaptive filtering technique.

\subsection{Discrete Wavelet Transform}

The Discrete Wavelet Transform (DWT) is discrete in time and scale, meaning that the DWT coefficients may have real (floating-point) values, but the time and scale values used to address these coefficients are numeral. The signal is partitioned by DWT into one or more levels of resolution (also called octaves), A 1-dimensional, one octave DWT. It comprises the analysis (wavelet transform) on the left side and the synthesis (inverse wavelet transform) on the right side. The low-pass filter produces the average signal, while the high pass filter produces the detail signal. In multi-resolution analysis, the average signal at one level is sent to an additional set of filters, which turns out the average and detail signals at the next octave [18]

\subsection{Dual Tree Complex Wavelet Transform}

Dual-tree Complex wavelet transform [16] is complex valued extension of the standard wavelet. Complex transform utilize complex valued filtering that partitioned the image into real and imaginary fraction in transform domain. The real and imaginary coefficients are used to compute magnitude and phase information. The key impetus for producing the dual-tree complex wavelet transform was shift invariance. The complex wavelets are capable to differentiate between positive and negative the diagonal sub-bands therefore DT-CWT improves the directional selectivity.

\subsection{Kernel Adaptive Filtering}

Kernel adaptive filtering is an adaptive filtering procedure for familiar nonlinear problems. It is a usual generalization of linear adaptive filtering in regenerating kernel Hilbert spaces. Kernel adaptive filters [17] are online kernel process, directly related to some artificial neural networks such as radial basis function networks and regularization networks. Some distinguishing attribute incorporates: The learning process is online, the learning method is convex which has no local minima, and the learning method requires moderate complexity.

The common updating rule of a kernel adaptive filter is

$\mathrm{f}_{\mathrm{i}}=\mathrm{f}_{\mathrm{i}-1}+\mathrm{g}(\mathrm{i}) \mathrm{e}(\mathrm{i})$

where $f_{i-1}$ is the estimate at time $i-1$. e (i) is the prediction error of $f_{i-1}$ on the ith data. The Kernel adaptive filters gives a new perspective for linear adaptive filters since linear adaptive filters become a special case being alternatively expressed in the dual space. Kernel adaptive filters clearly show that there is a growing memory structure embedded in the filter weights.
They naturally create a growing radial basis function network, learning the network topology and adapting the free parameters directly from data at the same time. The learning rule is a beautiful combination of the error-correction and memorybased learning, and potentially it will have a deep impact on our understanding about the essence of learning theory.

\subsection{Algorithm for Proposed Method}

1. Load an image and stored it in variable (I) then take dynamic histogram of the original image (I).

2. Now apply DWT transform on the selected image.

3. Now process it for analysis Dominant brightness level on the basis of the LL band of DWT process.

4. Since the model image is an unsigned 8-bit integer type (the most common situation), number of colors value will be 256 .

5. Including the decompositions on the basis of dominant brightness levels then proceed it.

- Approximation coefficient storage.

- Horizontal detail coefficient storage.

- Vertical detail coefficient storage.

- Diagonal detail coefficient storage.

6. Convert to unsigned 8-bit integer.

7. Including the Adaptive intensity transfer function on different intensity levels of the decomposed image and then smoothened out.

8. The smooth image is passed to the soble edge detection techniques which are then integrated with the Contrast enhancement techniques.

9. Now apply kernel function to filter an image after weighted map and smoothening edge process.

10. The inverse DWT is then applied on the fusion image and $\mathrm{HH}, \mathrm{HL}, \mathrm{LH}$ bands to get the contrasted image.

11. Now processed the all decomposed and layered images to fusion block to fuse all resultant images.

12. Then again apply the image histogram process block into the fused image.

13. Apply the histogram equalization of that image with the original image to get the enhanced image.

\section{RESULT ANALYSIS}

Here MATLAB2009A is used for the evolution of experimental result with 4GB RAM, I3 processor system configuration.

\subsection{Image Dataset}

The Computer Vision Group-University of Granada (CVGUGR) Database is online available at http://decsai.ugr.es/cvg/dbimagenes is very popular dataset. The website contains the various categorical dataset like it contains remote sensing dataset, medical image dataset, normal intensity dataset, color image dataset etc. The dataset are also available in the different dimension and according to the research interest area they can be downloaded without any cost. The images are aerial1.pgm, 7.1.01.pgm, 7.1.07.pgm, 42.pgm; srl2-dc.pgm respectively is used. In the figure 5.1.1 shows that the main GUI environment and the comparison of all three methods with proposed method. 


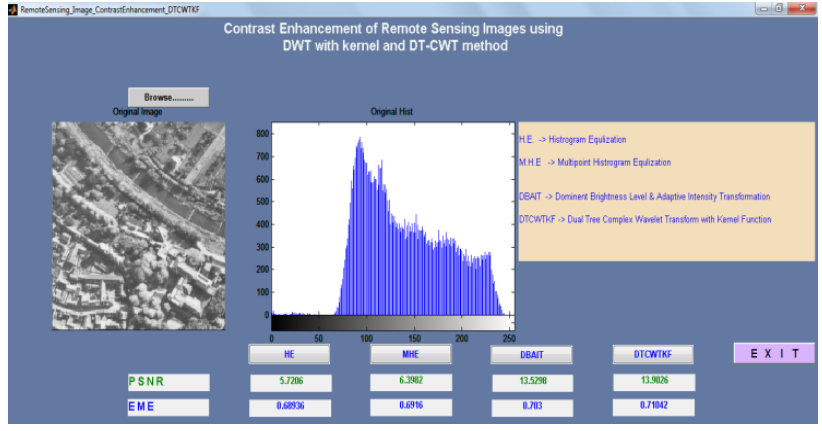

Figure 5.1.1: Result comparison of aerial1.pgm image

The GUI window the four methods are compared, and their PSNR, EME are shown bottom of the respective methods.

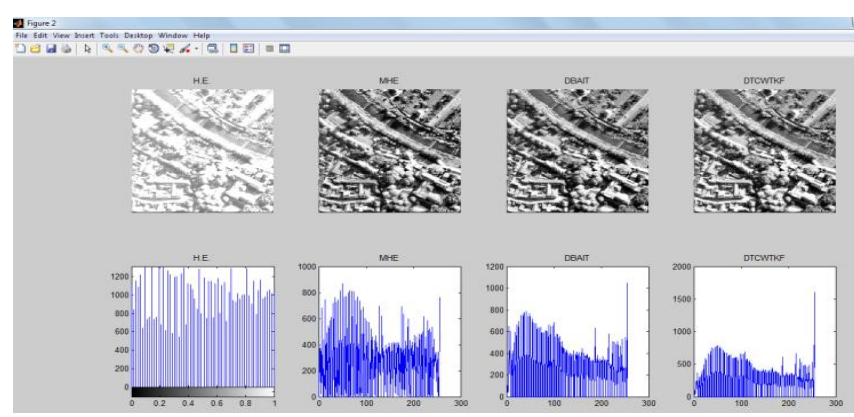

Figure 5.1.2: Histogram comparison of aerial1.pgm image

In the figure 5.1.2 shows comparisons of histogram and enhanced image result of the defined four methods. In the table 5.1.1 and table 5.1.2 shows the comparative analysis of PSNR and EME respectively after its comparison shown in figure 5.1.3 and figure 5.1.4 respectively.

Table 5.1.1: PSNR Comparison aerial1.pgm image

\begin{tabular}{|c|l|l|l|}
\hline HE & MHE & DBAIT & DTCWTKF \\
\hline 5.7206 & 6.3982 & 13.5298 & 13.9026 \\
\hline
\end{tabular}

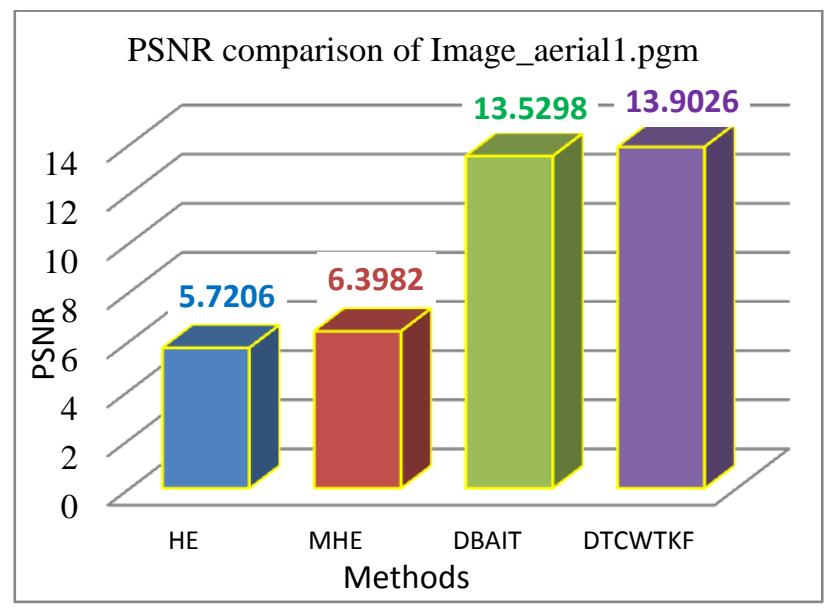

Figure 5.1.3: PSNR comparison of aerial1.pgm image
Table 5.1.2: EME Comparison aerial1.pgm image

\begin{tabular}{|c|l|l|l|}
\hline HE & MHE & DBAIT & DTCWTKF \\
\hline 0.68936 & 0.6916 & 0.703 & 0.71042 \\
\hline
\end{tabular}

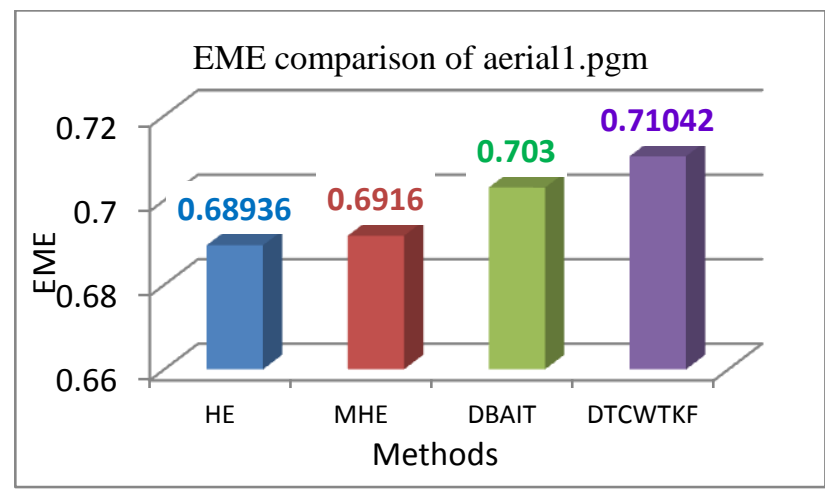

Figure 5.1.4: EME comparison of aerial1.pgg image

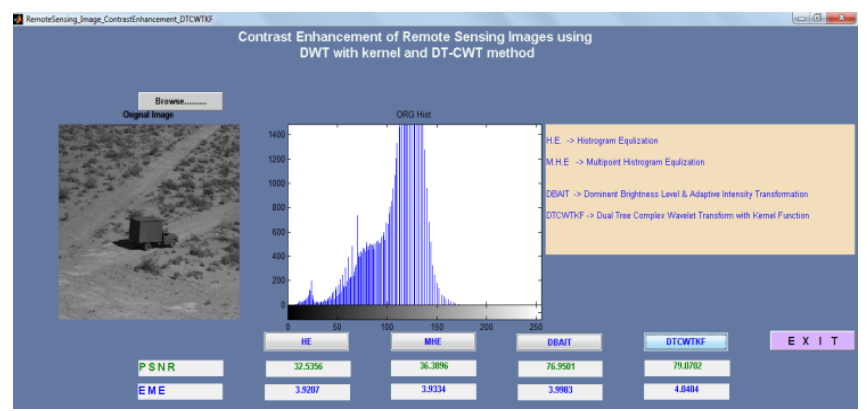

Figure 5.1.5: Result comparison of image_7.1.01.pgm

Here in the figure 5.1.5 is showing that the experimental comparison image_7.1.01.png image, where in the axes1 shows the original image, axes 2 shows its histogram. Then in the bottom of GUI window the four methods are compared, and their PSNR, EME are shown bottom of the respective methods.

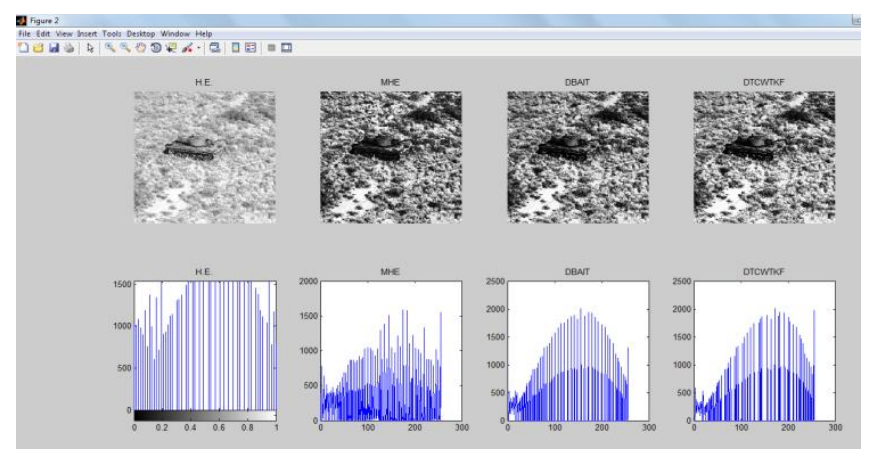

Figure 5.1.6: Histogram comparison of image 7.1.0.01.pgm

In the figure 5.1.6 shows comparisons of histogram and enhanced image result of defined four methods for image_7.1.01.png. In the table 5.1.3 and table 5.1.4 shows the comparative analysis of PSNR and EME respectively after its comparison, the comparison graph shown in figure 5.1.4 and figure 5.1.5 respectively. 
Table 5.1.3: PSNR Comparison image_7.1.01.pgm

\begin{tabular}{|c|c|c|c|}
\hline HE & MHE & DBAIT & DTCWTKF \\
\hline 32.5356 & 36.3896 & 76.9501 & 79.0702 \\
\hline
\end{tabular}

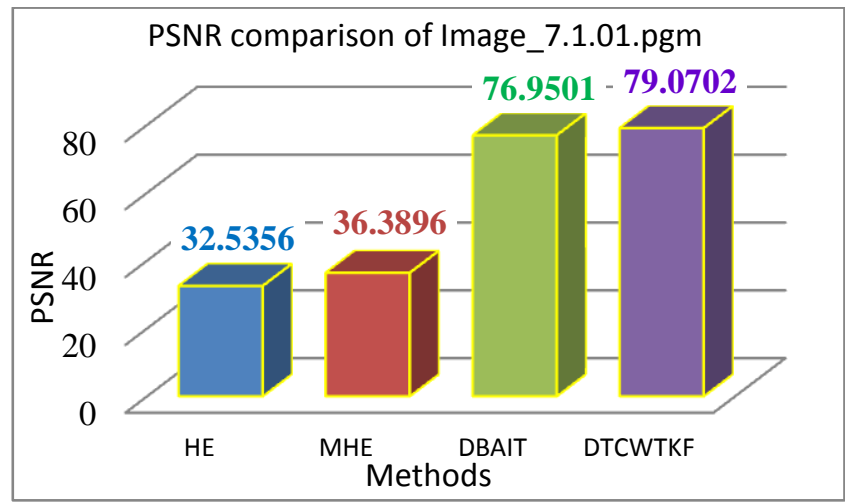

Figure 5.1.4: PSNR comparison of image_7.1.01.pgm

Table 5.1.4: EME Comparison image_7.1.01.pgm

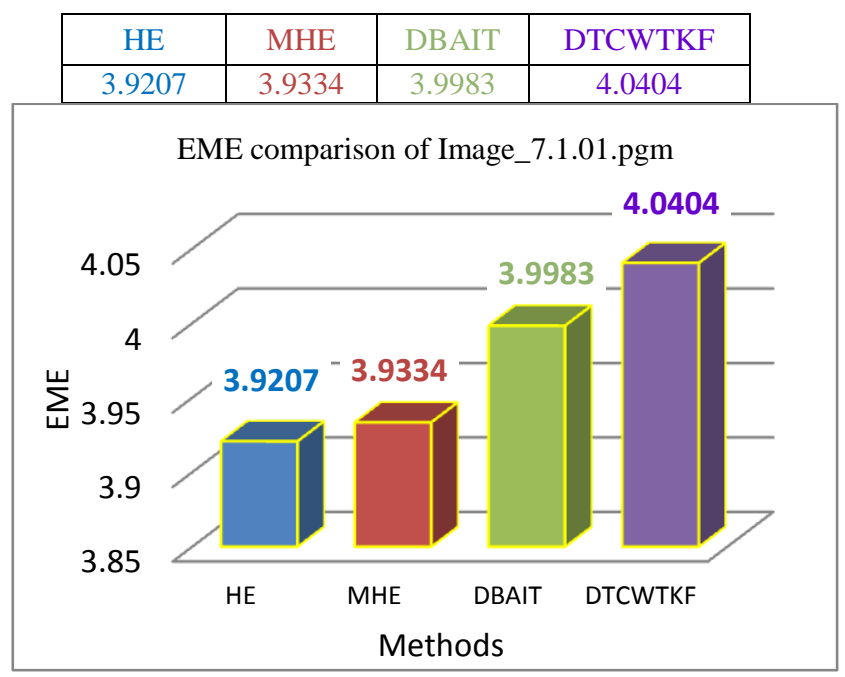

Figure 5.1.5: EME comparison of image_7.1.01.pgm

Similarly, we can compare the rest of images such as 7.1.07.pgm, 42.pgm, srl2-dc.pgm \& calculate their PSNR \& EME. After the detailed comparison of all methods with various different images, the overall performance of proposed DTCWTKF method is best in the comparison of both resultant parameters PSNR and EME. Now in the next section they are showing the summarized comparative result analysis of the all four methods.

Table 5.1.5: Summary and Comparison of PSNR

\begin{tabular}{|c|c|c|c|c|}
\hline Methods/Images & HE & MHE & DBAIT & DTCWTKF \\
\hline Image_42.pgm & $\mathbf{1 3 . 8 6 9 3}$ & $\mathbf{1 5 . 5 1 2 2}$ & 32.8024 & $\mathbf{3 3 . 7 0 6 2}$ \\
\hline Image_7.1.01.pgm & $\mathbf{3 2 . 5 3 5 6}$ & $\mathbf{3 6 . 3 8 9 6}$ & 76.9501 & $\mathbf{7 9 . 0 7 0 2}$ \\
\hline Image_7.1.07.pgm & $\mathbf{2 3 . 5 3 3 7}$ & $\mathbf{2 6 . 3 2 1 3}$ & 55.6595 & $\mathbf{5 7 . 1 9 3}$ \\
\hline Image_aerial1.pgm & $\mathbf{5 . 7 2 0 6}$ & $\mathbf{6 . 3 9 8 2}$ & $\mathbf{1 3 . 5 2 9 8}$ & $\mathbf{1 3 . 9 0 2 6}$ \\
\hline Image_srl2-dc.pgm & $\mathbf{5 . 9 9 6}$ & $\mathbf{6 . 7 0 6 2}$ & 14.1811 & $\mathbf{1 4 . 5 7 1 8}$ \\
\hline
\end{tabular}

Here table 5.1.5 shows that the overall PSNR performance of all four methods for the taken images so far. And the figure 5.1.6 shows the graph comparison analysis of the PSNR summary table.

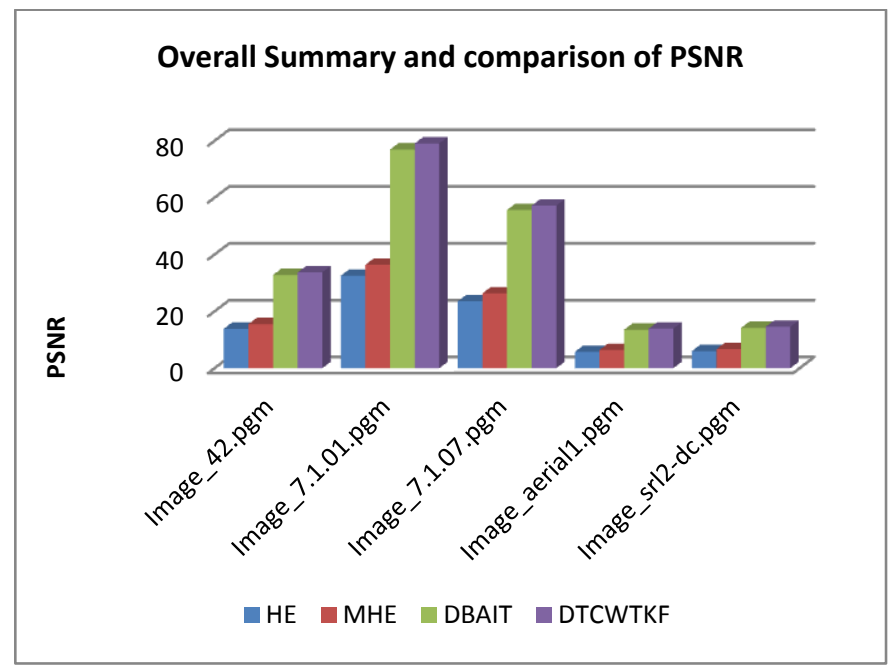

Figure 5.1.6: Overall Summary and comparison of PSNR

And here going to compare overall EME analysis of all four implemented methods with the help of summarized table and its comparison graph.

Table 5.1.6: Summary and Comparison of EME

\begin{tabular}{|c|c|c|c|c|}
\hline Methods/Images & HE & MHE & DBAIT & DTCWTKF \\
\hline Image_42.pgm & $\mathbf{1 . 6 7 1 3}$ & $\mathbf{1 . 6 7 6 7}$ & 1.7044 & $\mathbf{1 . 7 2 2 4}$ \\
\hline Image_7.1.01.pgm & $\mathbf{3 . 9 2 0 7}$ & $\mathbf{3 . 9 3 3 4}$ & 3.9983 & $\mathbf{4 . 0 4 0 4}$ \\
\hline Image_7.1.07.pgm & $\mathbf{2 . 8 3 5 9}$ & $\mathbf{2 . 8 4 5 1}$ & 2.892 & $\mathbf{2 . 9 2 2 5}$ \\
\hline Image_aerial1.pgm & $\mathbf{0 . 6 8 9 3 6}$ & $\mathbf{0 . 6 9 1 6}$ & 0.703 & $\mathbf{0 . 7 1 0 4 2}$ \\
\hline Image_srl2-dc.pgm & $\mathbf{0 . 7 2 2 5 4}$ & $\mathbf{0 . 7 2 8 4 9}$ & 0.73684 & $\mathbf{0 . 7 4 4 6 1}$ \\
\hline
\end{tabular}

Here table 5.1.6 shows that the overall EME performance of all four methods for taken images so far. And the figure 5.1.7 shows the graph comparison analysis of the EME summery table.

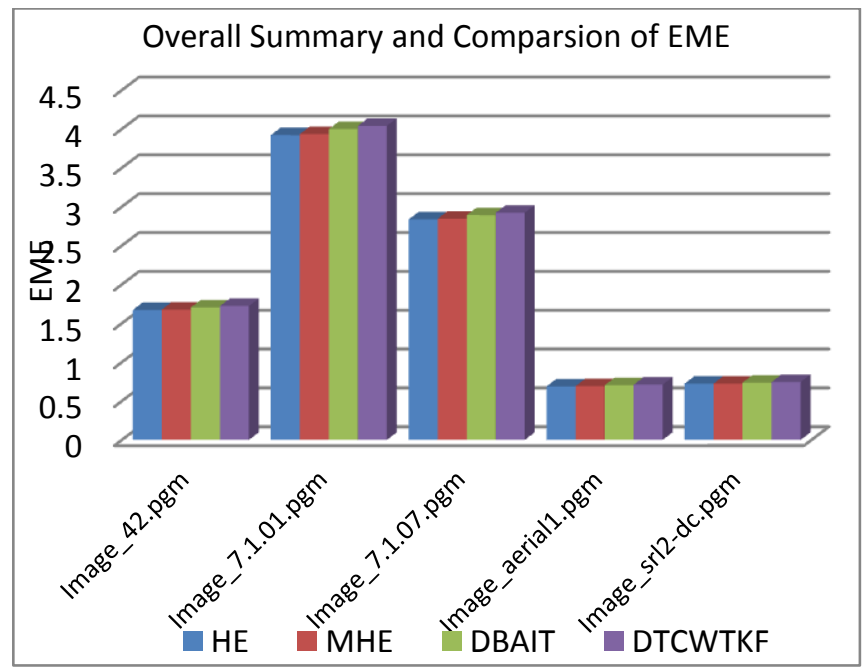

Figure 5.1.7: Overall Summary and comparison of EME 


\section{CONCLUSION AND FUTURE SCOPE}

For evaluating the performance of proposed method, we tested five low contrast remote sensing images taken from CVG-UGR image database. The performance of proposed algorithm compared with the existing well known algorithms including HE, MHE and DBAIT methods. And at overall analysis of the all compared algorithm the proposed method show the better result in all cases.

In digital image processing, here contrast image enhancement for remote sensing image data field a lot of work has been done to get better the quality of image such as histogram equalization, multi-histogram equalization and pixel dependent contrast preserving, Homomorphism filtering, Un-sharp Masking etc. Here in this the dissertation presents a proficient or effective technique of contrast enhancement using the concept of DWT with kernel adaptive filter. The proposed technique implemented here provides less error rate and have most Peak Signal to Noise Ration. This proposed method is an efficient as compared to the other existing techniques which doesn't provides more enhancement over contrast and also the brightness can't be preserved, but the technique implemented here not only improves the contrast enhancement but also the intensity can be maintained.

The technique implemented in this dissertation provides more contrast enhancement but when these concept can be used for the emissive display models then the power consumption is more, hence for the future work we provides an effective model for power displays such that the contrast of the image can be improved or get better, intensity gets preserved and more importantly power dissipation should be less.

\section{REFERENCES}

[1] Sasi Gopalan, Madhu S Nair and Souriar Sebastian "Approximation Studies on Image Enhancement Using Fuzzy Technique" International Journal of Advanced Science and Technology, Vol. 10, pp.11-26, September, 2009.

[2] S.S. Bedi, Rati Khandelwal "Various Image Enhancement Techniques- A Critical Review" International Journal of Advanced Research in Computer and Communication Engineering Vol. 2, Issue 3, March 2013

[3] Rakhi Chanana, Er.Parneet Kaur Randhawa, Er.Navneet Singh Randhawa "Spatial Domain based Image Enhancement Techniques for Scanned Electron Microscope (SEM) images" IJCSI International Journal of Computer Science Issues, Vol. 8, Issue 4, No 2, July 2011 ISSN (Online): 1694-0814

[4] Gonzalez, Rafael C.; Woods, Richard E. 2002. Digital Image Processing. Second Edition. Prentice Hall, New Jeresy. 2002, ISBN: 0-130-94650-8.

[5] Prof. Sumana Gupta. Digital image processing, Indian Institute of technology. [online]Published 2008. [citied1.5.2012].
[6] G. Veena, V. Uma, Ch. Ganapathy Reddy “Contrast Enhancement for Remote Sensing Images with Discrete Wavelet Transform", International Journal of Recent Technology and Engineering (IJRTE) ISSN: 2277-3878, Volume-2, Issue-3, July 2013

[7] Chi-Farn Chen, Hung-Yu Chang, Li-Yu Chang "A FuzzyBased Method For Remote Sensing Image Contrast Enhancement" The International Archives of the Photogrammetry, Remote Sensing and Spatial Information Sciences. Vol. XXXVII. Part B2. Beijing 2008

[8] Deepak Kumar Pandey, Rajesh Nema "Efficient Contrast Enhancement using Kernel Padding and DWT with Image Fusion" International Journal of Computer Applications (0975 - 8887) Volume 77- No.15, September 2013

[9] Hasan Demirel, Cagri Ozcinar, and Gholamreza Anbarjafari," Satellite Image Contrast Enhancement Using Discrete Wavelet Transform and Singular Value Decomposition", IEEE Geoscience and Remote Sensing Letters, vol. 7, no. 2, pp. 333-337, April 2010.

[10] Eunsung Lee, S.Kim, W.Kang, D.Seo and Jooki Paik "Contrast Enhancement using Domonant Brightness Level and Adaptive Intensity Transformation for Remote Sensing Image"IEEE Geoscience and Remote sensing letters, Vol. 10, no.1, January 2013

[11] Shujun Fu, Qiuqi Ruan, Wenqia Wang "Remote Sensing Image Data Enhancement Based on Robust Inverse Diffusion Equation for Agriculture Applications" ICSP 2008 Proceedings.

[12] Artur Łoza, David R. Bull, Paul R. Hill, Alin M .Achim "Automatic contrast enhancement of low-light images based on local statistics of wavelet coefficients", Digital Signal Processing (2013), www.elsevier.com/locate/dsp.

[13] Adin Ramirez Rivera, Byungyong Ryu, and Oksam Chae "Content Aware Dark Image Enhancement through Channel Divison"IEEE Transactions on Image Processing,volume 21,issue 9, 2012.

[14] S. E. Umbaugh, "Computer Vision \& Image Processing," Prentice Hall PTR, 1998.

[15] Seyed Mohammad Entezarmahdi,and Mehran Yazdi," Stationary Image Resolution Enhancement on the Basis of Contourlet and Wavelet Transforms by means of the Artificial Neural Network", 2010 IEEE.

[16] N. G. Kingsbury, "The dual-tree complex wavelet transform with improved orthogonality and symmetry properties", IEEE international Conference on Image processing, pages 375-378, September 2000.

[17] J. Kivinen, A. Smola and R. C. Williamson. Online learning with kernels, IEEE Transactions on Signal Processing, volume 52, issue 8, pages 2165-2176, 2004.

[18] S. Mallat, "A Theory for Multiresolution Signal Decomposition: The Wavelet Representation," IEEE Pattern Analysis 